\title{
Comorbid anxiety and depression in childhood was associated with poorer psychosocial functioning in early adulthood
}

\author{
Last CG, Hansen CH, Franco N.Anxious children in adulthood: a prospective study of adjustment. J Am Acad Child Adolesc Psychiatry \\ 1997 May;36:645-52.
}

\section{Objective}

To determine the effect of childhood anxiety disorders (with and without depression) on psychosocial functioning in early adulthood and to identify predictors of outcome.

\section{Design}

Cohort analytic study with cases and controls matched on age and sex \{with 8 to 10 years of follow up\}*.

\section{Setting}

Pittsburgh, USA.

\section{Participants}

62 participants (aged $>18$ y) who had previously participated in a study of childhood anxiety disorders and who agreed to be interviewed by telephone. Between the ages of 5 and 17 years, participants had been diagnosed as having an anxiety disorder, with or without comorbid depression, according to the Diagnostic and Statistical Manual of Mental Disorders, 3rd edition, revised. 39 participants without a history of psychiatric illness were recruited from the community.

\section{Assessment of risk factors}

Participants were classified as anxious, anxious and depressed, or never psychiatrically ill based on their diagnosis in the childhood study. Telephone interviews were used to gather data on psychosocial functioning, sociodemographic, and clinical variables. Possible predictors of adult adjustment included type and severity of diagnosis, number of anxiety diagnoses, sex, age of onset, and parental psychopathology.

\section{Main outcome measures}

Main outcome measures included current psychological func- tioning, use of mental health services, occupational and school functioning, and residence.

\begin{abstract}
Main results
Participants who were anxious and depressed as children reported more psychological problems $(46 \%)$ in the past 5 years than either those who were only anxious in childhood $(12 \%$, $\mathrm{p}<0.01)$ or those without a history of psychiatric illness $(13 \%$, $\mathrm{p}<0.01)$. Furthermore, participants who were anxious and depressed reported using more mental health services $(25 \%)$ than those who were only anxious $(3 \%, \mathrm{p}<0.05)$. Participants classified as anxious and depressed were also less likely to be employed or in school $(75 \%)$ than participants with no history of psychiatric illness $(95 \%, \mathrm{p}<0.05)$. Participants with only an anxiety disorder were less likely to live independently (24\%) than participants with no history of psychiatric illness $(51 \%, \mathrm{p}<0.05)$. None of the child or parent variables was a statistically significant predictor of psychosocial functioning.
\end{abstract}

\section{Conclusions}

Young adults with a childhood diagnosis of anxiety showed similar psychosocial adjustment to those without a history of psychiatric illness except in the area of independent living. Young adults with a childhood diagnosis of comorbid anxiety and depression had poorer psychosocial functioning than those without a history of psychiatric illness or with a childhood diagnosis of anxiety disorder only. Sociodemographic and clinical variables did not predict adult adjustment for adults with a history of anxiety disorders.

Source of funding: in part, National Institute of Mental Health.

For article reprint:Dr C G Last, 3111 University Drive, Suite 307, Coral Springs, FL 33065, USA. Fax +19542623857

*Information on follow up provided by the author.

\section{Commentary}

This study by Last et al provides a rare look into the long term effect of early onset anxiety disorders on the social and psychological functioning of young adults. In considering the aspects of functioning addressed in this study an important distinction emerges between the diagnostic course (most commonly characterised by a number of "snap shot" views of symptom counts and criteria across development) and the associated or resulting functional impairment. This distinction is often blurred due to the fact that impairment is an integral part of diagnostic criteria. Typically, impairment becomes subsumed under the more general discussion of the phenomenology of psychiatric disorders, rather than taking its place as an important and distinct measure of outcome.

Last $e t$ al take a first step towards disen- tangling these related but separate constructs by focusing on a number of normative aspects of functioning that mark the transition into young adulthood. While their findings indicate the substantial influence of comorbid anxiety and depression on various aspects of functioning, it is more important to note that adults in the anxious and depressed group were also more likely to report psychological problems within the previous 5 years. This suggests that characteristics of the diagnostic course-that is, recurrent or chronic symptoms-may mediate the association between early onset psychopathology and the functional impairment seen in early adulthood.

Recent prospective work by Klein et al has highlighted the added utility of examining individuals with childhood onset disorders whose symptoms have remitted in late adolescence or early adulthood. This complementary approach can be used to disentangle the residual impairment experienced after symptoms have remitted from the functional impairment caused by the recurrence or chronicity of the disorder. These distinctions have particularly important implications for clinicians who treat adolescents and young adults with continued functional impairment despite a partial or complete recovery from symptoms.

Lisa C Dierker, $\mathrm{PhD}$ Wesleyan University Middletown, Connecticut, USA

1 Klein DN, Lewinsohn PM, Seeley JR. Psychosocial characteristics of adolescents with a past history of dysthymic disorder: comparison with adolescents with past histories of major depressive and non-affective disorders, and never mentally ill controls. I Affect Disord 1997;42:127-35. 